\title{
Exact Controllability of an Impulsive Semilinear System with Deviated Argument in a Banach Space
}

\author{
Sanjukta Das, Dwijendra N. Pandey, and N. Sukavanam \\ Department of Mathematics, IIT Roorkee, Roorkee, Uttarakhand 247667, India \\ Correspondence should be addressed to Sanjukta Das; sanjukta_das44@yahoo.com
}

Received 28 April 2014; Accepted 30 June 2014; Published 16 July 2014

Academic Editor: Honglei Xu

Copyright (C) 2014 Sanjukta Das et al. This is an open access article distributed under the Creative Commons Attribution License, which permits unrestricted use, distribution, and reproduction in any medium, provided the original work is properly cited.

\begin{abstract}
A functional differential equation with deviated argument coupled with impulsive conditions is studied for the existence and uniqueness of the mild solution and exact controllability of the system. The results are obtained by using Banach contraction principle and $C_{0}$ semigroup theory without imposing additional assumptions such as analyticity and compactness conditions on the generated semigroup and the nonlinear term. An example is provided to illustrate the presented theory.
\end{abstract}

\section{Introduction}

It is possible to steer various dynamical systems from an arbitrary initial state to final state using the set of admissible controls. Such systems are called controllable. The theory for abstract linear control systems in finite-dimensions is well established. Many authors have extended the theory to infinite dimensional systems represented by nonlinear evolution equations. References of which are available in various articles such as [1-4].

As the change of state occurs abruptly in several physical phenomena, many authors have discussed impulsive differential equations in their articles. For instance, Chang [5] and Li et al. [6] discussed the controllability of impulsive functional differential system in Banach spaces using Schaefer's fixed point theorem. Jeong et al. [7] investigated the controllability of semilinear retarded control systems in Hilbert spaces. Tai and Wang [8] proposed sufficient conditions for the controllability of fractional impulsive neutral functional integrodifferential systems in a Banach space. Sakthivel and Anandhi [9] discussed approximate controllability of impulsive differential equations with state dependent delay.

Controllability results are available in overwhelming majority for abstract impulsive differential systems rather than for impulsive functional differential equation with deviated arguments.
As demand for efficient dynamic performances increase, mathematical models are required to behave more like real processes. Models are designed to deal with aftereffect, hereditary systems, equations with deviated arguments, and so forth. Delay differential systems are still resistant to quite a number of "classical controllers." Many partial differential systems can be reduced to functional differential equations with deviated arguments. Thus controllability of functional differential equation with deviated argument has to be extensively studied. However, Gal [10] studied the existence and uniqueness of local and global solutions for initial value problem with deviated argument

$$
\begin{array}{r}
u^{\prime}(t)=A u(t)+f(t, u(t), u[\alpha(u(t), t)]), \\
t \in R_{+}, \quad u(0)=u_{0} .
\end{array}
$$

Pandey et al. [11] used analytic semigroup theory and fixed point arguments to study the existence and uniqueness of mild solutions of a neutral differential equation with a deviated argument in a Banach space.

Chang and Liu [12] discussed the existence of mild and strong solutions for some neutral partial differential equations with nonlocal conditions using Sadovskii fixed point theorem and compact analytic semigroup of uniformly bounded linear operators. 
Fractional operators, analyticity, and compactness are mostly used to establish these results which impose more restriction on the semigroup and the nonlinear part of the semilinear system. Interestingly controllability results for functional differential equation with deviated argument coupled with impulsive conditions are not widely available so far.

In this work the exact controllability of a semilinear system governed by an impulsive functional differential equation is studied. The semilinear system is illustrated in Section 2 as an abstract differential equation with deviated argument and impulsive conditions. Impulsive conditions reflect abrupt changes in real physical phenomenon that are more interesting than initial conditions. In Section 3 simple Lipschitz conditions and $C_{0}$ semigroup theory is required to study the differential equation with deviated argument removing the more strict assumptions like analyticity and compactness of the semigroup or the nonlinear part.

\section{Preliminaries}

In this section some basic definitions and lemmas are stated. Naito [13] and Pazy [14] illustrated the basic concepts of controllability and semigroup theory, respectively.

Let $J=[0, T], 0<t_{1}<t_{2}<\cdots<t_{m}<T$ and $J^{\prime}=[0, T] \backslash$ $\left\{t_{1}, t_{2}, \ldots, t_{m}\right\} \subset J$. Let $X$ and $U$ be Banach spaces. The complete controllability of the following impulsive differential equation is studied. Consider

$$
\begin{gathered}
\frac{d}{d t} x(t)=A x(t)+f(t, x(t), x(a(x(t), t)))+B u(t), \\
t \in J^{\prime}, \\
x(0)=x_{0}, \\
\Delta x\left(t_{k}\right)=I_{k}\left(x\left(t_{k}\right)\right), \quad k=1,2, \ldots, m, \\
0<t_{1}<\cdots<t_{m}<T .
\end{gathered}
$$

The state function $x(\cdot)$ takes values in Banach space $X$. The control function $u(\cdot) \in L^{2}(J, U)$, where $U$ is a Banach space. $B: U \rightarrow X$ is a bounded linear operator. $A: D(A) \subset X \rightarrow$ $X$ is a the infinitesimal generator of a strongly continuous semigroup $S(t), t>0$ on $X . f$ and $a$ are suitably defined functions satisfying certain conditions to be specified in Section 3. $I_{k} \in C(X, X), k=1,2, \ldots, m$ and $\Delta x\left(t_{k}\right)=x\left(t_{k}^{+}\right)-$ $x\left(t_{k}^{-}\right)=x\left(t_{k}^{+}\right)-x\left(t_{k}\right)$.

We define $P C(J, X)=\left\{x:[0, T] \rightarrow X \mid x \in C\left(J^{\prime}, X\right)\right.$, $x\left(t_{k}^{+}\right)$and $x\left(t_{k}^{-}\right)$exists, and $\left.x\left(t_{k}^{-}\right)=x\left(t_{k}\right), 1 \leq k \leq m\right\}$.

$P C(J, X)$ is a Banach space with norm $\|x\|=\sup _{t \in J}\|x(t)\|$.

Let $D=C_{L}(J, X)=\{u \in P C(J, X):\|u(t)-u(s)\| \leq$ $\left.L|t-s|, \forall t, s \in J^{\prime}\right\}$.

Definition 1 (see [13]). System (2) is said to be controllable on the interval $J$ if for every $x_{0}, x_{T} \in X$, there exists a control $u \in$ $L^{2}(J, U)$ such that the mild solution of (2) satisfies $x(0)=x_{0}$, $x(T)=x_{T}$.
Definition 2. A mild solution of problem (2) is defined as function $x: J \rightarrow D$ such that it satisfies the following:

(i) $x(0)=x_{0}$,

(ii) $\Delta x\left(t_{k}\right)=I_{k}\left(x\left(t_{k}\right)\right), k=1,2, \ldots, m$,

(iii) the restriction of $x(\cdot)$ to the interval $J^{\prime}$ is continuous and the following integral equation is satisfied:

$$
\begin{aligned}
x(t)= & S(t) x_{0} \\
& +\int_{0}^{t} S(t-s)[f(s, x(s), x(a(x(s), s))) \\
& +(B u)(s)] d s \\
& +\sum_{0<t_{k}<t} S\left(t-t_{k}\right) I_{k}\left(x\left(t_{k}\right)\right)
\end{aligned}
$$

where $S(t)$, for all $t \in J$, denotes the $C_{0}$ semigroup generated by $A$.

Throughout this paper, the following assumptions are made. Let

$$
Z_{k}=\{x(t) \in D:\|x(t)\| \leq k\}
$$

(H1) A generates a strongly continuous semigroup $S(t)$ in the Banach space $X$ and there exists a constant $M$ such that $\|S(t)\| \leq M$.

(H2) The nonlinear operator $f: J \times X \times X \rightarrow X$ and satisfy the Lipschitz condition such that $\exists$ constants $M_{1}, M_{2}>0$ such that for all $x_{i} \in Z_{k}, y_{i} \in Z_{k}, i=1,2$, we have

$$
\begin{aligned}
& \left\|f\left(t, x_{1}, y_{1}\right)-f\left(t, x_{2}, y_{2}\right)\right\| \\
& \quad \leq M_{1}\left\{\left\|x_{1}-x_{2}\right\|+\left\|y_{1}-y_{2}\right\|\right\}
\end{aligned}
$$

and $\max _{t \in J}\|f(t, 0, x(0))\|=M_{2}$.

(H3) Moreover assume $a: D \times \mathbb{R}_{+} \rightarrow \mathbb{R}_{+}$is globally continuous on $X \times \mathbb{R}_{+}$and satisfies $a(\cdot, 0)=0$ and for each $\delta>0$, there is a $L_{a}=L_{a}(\delta)>0$ such that $|a(u, t)-a(v, t)| \leq L_{a}\|u-v\|, \forall u, v \in X$, whenever $0 \leq t \leq \delta$.

(H4) The linear operator $W: L_{2}[J, U] \rightarrow X$, defined by

$$
W u=\int_{0}^{T} S(T-s) B u(s) d s
$$

has an invertible operator $W^{-1}$ (induced by $W$ ) defined on $L_{2}[J, U] / \operatorname{Ker} W$ and there exist positive constants $K_{1}>0, K_{2}>0$ such that $\left\|W^{-1}\right\| \leq K_{1}$ and $\|B\| \leq K_{2}$.

(H5) There exist constants $d, L_{k}, I, d_{k}>0, k=1, \ldots, m$, such that $\left\|I_{k}(\cdot)\right\|<d_{k}, \sum_{k=1}^{m} d_{k}=d,\left\|I_{k}(x)-I_{k}(y)\right\| \leq$ $L_{k}\|x-y\| \forall x, y \in X$, and $\sum_{k=1}^{m} L_{k}=I$. 


\section{Main Result}

In this section we discuss the existence and uniqueness of mild solution and exact controllability of the semilinear differential equation with deviated argument and impulsive conditions described in Section 2.

Now,

$$
\begin{aligned}
M[ & \left\|x_{0}\right\|+T K_{1} K_{2}\left\|x_{T}\right\| \\
& +M M_{2} K_{1} K_{2} T^{2}+T M K_{1} K_{2}\left\|x_{0}\right\| \\
& +M M_{1} K_{1} K_{2} T^{2}\left(k+L^{2} L_{a} T\right) \\
& +2 M_{1} T\left(k+L^{2} L_{a} T\right)+M_{2} T \\
& \left.+\left(1+M K_{1} K_{2} T\right) d\right] \leq k,
\end{aligned}
$$

where $k>0$ is a constant. Let

$$
p=\left[\left(2+L L_{a}\right)\left(M K_{1} K_{2} T+1\right) M M_{1} T+M I+M^{2} K_{1} K_{2} T I\right]
$$

be such that $0 \leq p<1$.

Theorem 3. If hypotheses (H1)-(H5) and (7) and (8) are satisfied then system (2) is controllable on J.

Proof. Let $x \in Z_{k}$. Using hypothesis (H4) for an arbitrary function $x(\cdot)$,we define the control

$$
\begin{aligned}
u(t)=W^{-1}[ & x_{T}-S(T) x_{0} \\
& -\int_{0}^{T} S(T-s) f(s, x(s), x(a(x(s), s))) d s \\
& \left.-\sum_{k=1}^{m} S\left(T-t_{k}\right) I_{k}\left(x\left(t_{k}\right)\right)\right](t) .
\end{aligned}
$$

Using this control and Banach contraction principle, the operator defined by

$(\Phi x)(t)$

$$
\begin{aligned}
= & S(t) x_{0} \\
& +\int_{0}^{t} S(t-s)[(B u)(s)+f(s, x(s), x(a(x(s), s)))] d s \\
& +\sum_{0<t_{k}<t} S\left(t-t_{k}\right) I_{k}\left(x\left(t_{k}\right)\right)
\end{aligned}
$$

is proved to have a fixed point; that is, $(\Phi x)(T)=x_{T}$. This fixed point is then a solution of (2). This implies that $\exists$ the control $u$ which steers the semilinear system from the initial state $x_{0}$ to $x_{T}$ in time $T$ provided we can obtain a fixed point of the nonlinear operator $\Phi$. First we prove $\Phi$ maps $Z_{k}$ into itself. For this, using conditions (H1)-(H5), (4), (9), (7), and triangle inequality, we have

$$
\begin{aligned}
& \|(\Phi(x))(t)\| \\
& \leq\left\|S(t) x_{0}\right\| \\
& +\| \int_{0}^{t} S(t-s) B u(s) d s \\
& +\int_{0}^{t} S(t-s) f(s, x(s), x(a(x(s), s))) d s \| \\
& +\left\|\sum_{0<t_{k}<t} S\left(t-t_{k}\right) I_{k}\left(x\left(t_{k}\right)\right)\right\| \\
& =\left\|S(t) x_{0}\right\|
\end{aligned}
$$

$+\| \int_{0}^{t} S(t-s) B W^{-1}$

$$
\times\left[x_{T}-S(T) x_{0}\right.
$$$$
-\int_{0}^{T} S(T-\eta) f(\eta, x(\eta), x(a(x(\eta), \eta))) d \eta
$$$$
\left.+\sum_{k=1}^{m} S\left(T-t_{k}\right) I_{k}\left(x\left(t_{k}\right)\right)\right](s) d s
$$$$
+\int_{0}^{t} S(t-s) f(s, x(s), x(a(x(s), s))) d s \|
$$

$$
+\left\|\sum_{0<t_{k}<t} S\left(t-t_{k}\right) I_{k}\left(x\left(t_{k}\right)\right)\right\|
$$

$$
\begin{aligned}
& <\left\|S(t) x_{0}\right\| \\
& +\int_{0}^{t}\|S(t-s)\|\|B\|\left\|W^{-1}\right\| \\
& \quad \times \|\left[x_{T}-S(T) x_{0}\right. \\
& \quad-\int_{0}^{T} S(T-\eta) f(\eta, x(\eta), x(a(x(\eta), \eta))) d \eta \\
& \left.\quad+\sum_{k=1}^{m} S\left(T-t_{k}\right) I_{k}\left(x\left(t_{k}\right)\right)\right](s) d s \| \\
& +\left\|\int_{0}^{t} S(t-s) f(s, x(s), x(a(x(s), s))) d s\right\| \\
& +M \sum_{k=1,2, \ldots, m} d_{k}
\end{aligned}
$$




$$
\begin{aligned}
& \leq M\left\|x_{0}\right\|+M K_{1} K_{2} \\
& \times \int_{0}^{t}\left[\left\|x_{T}\right\|+M\left\|x_{0}\right\|\right. \\
& +M \sum_{k=1,2, \ldots m} d_{k} \\
& +M \int_{0}^{T}\{\| f(\eta, x(\eta), x(a(x(\eta), \eta))) \\
& -f(\eta, 0, x(0)) \| \\
& +\|f(\eta, 0, x(0))\|\} d \eta] d s \\
& +M \int_{0}^{t}\{\| f(s, x(s), x(a(x(s), s))) \\
& -f(s, 0, x(0)) \| \\
& +\|f(s, 0, x(0))\|\} d s+M d \\
& \leq M\left\|x_{0}\right\|+M K_{1} K_{2} \\
& \times \int_{0}^{t}\left[\left\|x_{T}\right\|+M\left\|x_{0}\right\|+M d\right. \\
& +M M_{1} \int_{0}^{T}\{\|x(\eta)\| \\
& +\|x(a(x(\eta), \eta))-x(a(x(0), 0))\|\} d \eta \\
& \left.+M M_{2} T\right] d s \\
& +M M_{1} \int_{0}^{t}\{\|x(s)\|+\|x(a(x(s), s))-x(a(x(0), 0))\|\} d s \\
& +M M_{2} T+M d \\
& \leq M\left\|x_{0}\right\|+M K_{1} K_{2} T\left\|x_{T}\right\|+M^{2} K_{1} K_{2} T\left\|x_{0}\right\| \\
& +M^{2} M_{1} K_{1} K_{2} T^{2}\left(k+L^{2} L_{a} T\right) \\
& +M^{2} M_{2} K_{1} K_{2} T^{2}+M^{2} K_{1} K_{2} T d \\
& +M M_{1} T\left(k+L^{2} L_{a} T\right)+M M_{2} T+M d \\
& \leq M\left[\left\|x_{0}\right\|+T K_{1} K_{2}\left\|x_{T}\right\|+T M K_{1} K_{2}\left\|x_{0}\right\|\right. \\
& +M M_{1} K_{1} K_{2} T^{2}\left(k+L^{2} L_{a} T\right) \\
& +M M_{2} K_{1} K_{2} T^{2}+2 M_{1} T\left(k+L^{2} L_{a} T\right) \\
& \left.+M_{2} T+\left(1+M K_{1} K_{2} T\right) d\right]
\end{aligned}
$$$$
\leq k \text {. }
$$

Therefore by hypothesis (H5), $\|(\Phi x)(t)\| \leq k$, which proves $\Phi\left(Z_{k}\right) \subseteq Z_{k}$. Thus $\Phi$ maps $Z_{k}$ into itself. Next $\Phi$ is proved to be a contraction map. Let $x_{1}, x_{2} \in Z_{k}$. Then

$$
\begin{aligned}
& \left\|\left(\Phi x_{1}\right)(t)-\left(\Phi x_{2}\right)(t)\right\| \\
& =\| \int_{0}^{t} S(t-s) B W^{-1} \\
& -\left[\int_{0}^{T} S(T-\eta)\right. \\
& \times\left\{f\left(\eta, x_{2}(\eta), x_{2}\left(a\left(x_{2}(\eta), \eta\right)\right)\right)\right. \\
& \left.-f\left(\eta, x_{1}(\eta), x_{1}\left(a\left(x_{1}(\eta), \eta\right)\right)\right)\right\} d \eta \\
& \left.+\left\|\sum_{k=1}^{m} S\left(T-t_{k}\right)\left[I_{k}\left(x_{2}\left(t_{k}\right)\right)-I_{k}\left(x_{1}\left(t_{k}\right)\right)\right]\right\|\right](s) d s \\
& +\int_{0}^{t} S(t-s) \\
& \times\left\{f\left(s, x_{2}(s), x_{1}\left(a\left(x_{2}(s), s\right)\right)\right)\right. \\
& \left.-f\left(s, x_{1}(s), x_{2}\left(a\left(x_{1}(s), s\right)\right)\right)\right\} d s \| \\
& +\left\|\sum_{0<t_{k}<t} S\left(t-t_{k}\right)\left[I_{k}\left(x_{2}\left(t_{k}\right)\right)-I_{k}\left(x_{1}\left(t_{k}\right)\right)\right]\right\| \\
& \leq M K_{1} K_{2} \\
& \times \int_{0}^{t}\left[M M_{1}\right. \\
& \times \int_{0}^{T}\left\{\left\|x_{2}(\eta)-x_{1}(\eta)\right\|\right. \\
& +\| x_{2}\left(a\left(x_{2}(\eta), \eta\right)\right) \\
& \left.-x_{1}\left(a\left(x_{1}(\eta), \eta\right)\right) \|\right\} d \eta \\
& \left.+M \sum_{k=1}^{m} L_{k}\left\|x_{1}-x_{2}\right\|\right] d s \\
& +M M_{1} \int_{0}^{t}\left[\left\|x_{1}(s)-x_{2}(s)\right\|\right. \\
& +\| x_{1}\left(a\left(x_{1}(s), s\right)\right) \\
& \left.-x_{2}\left(a\left(x_{2}(s), s\right)\right) \|\right] d s \\
& +M \sum_{k=1,2 \ldots, m} L_{k}\left\|x_{2}-x_{1}\right\| \\
& \leq M^{2} M_{1} K_{1} K_{2} T^{2} \\
& \times\left[\left\|x_{2}-x_{1}\right\|\right. \\
& +\left\|x_{2}\left(a\left(x_{2}(\eta), \eta\right)\right)-x_{1}\left(a\left(x_{2}(\eta), \eta\right)\right)\right\| \\
& \left.+\left\|x_{1}\left(a\left(x_{2}(\eta), \eta\right)\right)-x_{1}\left(a\left(x_{1}(\eta), \eta\right)\right)\right\|\right] \\
& +M^{2} K_{1} K_{2} T I\left\|x_{1}-x_{2}\right\| \\
& +M M_{1} T\left[\left\|x_{2}-x_{1}\right\|\right.
\end{aligned}
$$




$$
\begin{aligned}
& +\left\|x_{2}\left(a\left(x_{2}(s), s\right)\right)-x_{1}\left(a\left(x_{2}(s), s\right)\right)\right\| \\
& \left.+\left\|x_{1}\left(a\left(x_{2}(s), s\right)\right)-x_{1}\left(a\left(x_{1}(s), s\right)\right)\right\|\right] \\
& +M I\left\|x_{2}-x_{1}\right\| \\
& \leq M^{2} M_{1} K_{1} K_{2} T^{2}\left[\left(2+L L_{a}\right)\left\|x_{2}-x_{1}\right\|\right] \\
& +M^{2} K_{1} K_{2} T I\left\|x_{1}-x_{2}\right\| \\
& +M M_{1} T\left[\left(2+L L_{a}\right)\left\|x_{2}-x_{1}\right\|\right] \\
& +M I\left\|x_{2}-x_{1}\right\| \\
& \leq\left[\left(2+L L_{a}\right)\left(M K_{1} K_{2} T+1\right) M M_{1} T\right. \\
& \left.\quad+M I+M^{2} K_{2} K_{1} T I\right]\left\|x_{2}-x_{1}\right\| \\
& \leq p\left\|x_{1}-x_{2}\right\| .
\end{aligned}
$$

By applying conditions (H2), (H3), (H5), and (8) we find $\Phi$ is a contraction mapping on $Z_{k}$. Hence there exists a unique fixed point $x \in Z_{k}$ such that $(\Phi x)(t)=x(t)$. The unique fixed point of $\Phi$ is a mild solution of (2) on $J$, which satisfies $x(T)=$ $x_{T}$. Hence the system is controllable on $J$.

\section{Example}

Consider the following third order dispersion equation:

$$
\begin{aligned}
z_{t}(t, x)= & \frac{\partial^{3}}{\partial x^{3}} z(x, t)+B u(t) \\
& +p(t, z(x, t), z(a(z(x, t), t))) \\
\Delta z\left(t_{i}, x\right)= & I_{k}\left(z\left(t_{i}, x\right)\right), \quad t_{i} \in J^{\prime}, i=1, \ldots, m
\end{aligned}
$$

and the given initial and boundary conditions

$$
\frac{\partial^{k}}{\partial x^{k}} z(0, t)=\frac{\partial^{k}}{\partial x^{k}} z(2 \pi, t)=0, \quad k=0,1,2 ;
$$

$x \in I=(0,2 \pi), t \in J=[0, T]$.

Here $B: U \rightarrow X$ is a linear operator such that there exists an inverse operator $W^{-1}$ on $L^{2}(J ; U) / \operatorname{Ker} W$, where $W$ is defined by

$$
W u=\int_{0}^{T} S(T-s) B u(s) d s .
$$

The operator $S(t)$ is uniformly bounded and $p: J \times X \times X \rightarrow$ $X$ is continuous and uniformly bounded.

This problem can be brought to the form (2) by making suitable choices of $A, B$, and $f$.

Let $U=L^{2}[0,2 \pi], X=\left\{x \in L^{2}[0,2 \pi]:[x]=\right.$ $\left.\int_{0}^{2 \pi} x(s) d s=0\right\}, A w=-w_{x x x}$, and $D(A)=\left\{w \in H^{3}(0,2 \pi):\right.$ $\left.\left(\partial^{k} / \partial x^{k}\right) w(0)=\left(\partial^{k} / \partial x^{k}\right) w(1), k=0,1,2\right\}$.

Let $f(t, w, w(a(w, t)))=p(t, w(x), w(a(w(x), t)))$ for all $(t, w, w(a(w, t))) \in J \times X \times X, x \in I$ be such that condition (H2), (H3), and (H5) are satisfied.
Then this system becomes an abstract formulation of the type (2). The solutions are all bounded.

Further, all the conditions stated in the above theorem are satisfied. Hence system is exactly controllable on $J$.

\section{Conclusion}

Impulsive semilinear system of type (2) with deviated argument is studied, subsequently proving the sufficient conditions for exact controllability. Only $C_{0}$ semigroup is used throughout the paper. Our approach eliminates the need to assume the conditions such as analyticity and compactness of the semigroup and the function $f$. Banach contraction mapping principle is used to prove that the unique fixed point exists which is the mild solution of (2). Hence the system (2) is proved to be controllable.

\section{Conflict of Interests}

The authors declare that there is no conflict of interests regarding the publication of this paper.

\section{Acknowledgments}

The authors would like to express their sincere gratitude to the reviewers for their valuable suggestions. The first author would like to thank Ministry of Human Resource and Development with Grant no. MHR-02-23-200-429/304 for their funding.

\section{References}

[1] J. P. Dauer and N. I. Mahmudov, "Controllability of some nonlinear systems in Hilbert spaces," Journal of Optimization Theory and Applications, vol. 123, no. 2, pp. 319-329, 2004.

[2] K. Balachandran and E. R. Anandhi, "Boundary controllability of integrodifferential systems in Banach spaces," Proceedings of the Indian Academy of Sciences A: Mathematical Sciences, vol. 111, no. 1, pp. 127-135, 2001.

[3] K. Balachandran and J. Y. Park, "Existence of solutions and controllability of nonlinear integrodifferential systems in Banach spaces," Mathematical Problems in Engineering, vol. 2003, no. 12, pp. 65-79, 2003.

[4] N. Sukavanam and Divya, "Exact and Approximate controllability of abstract semilinear control systems," Indian Journal of Pure and Applied Mathematics, vol. 33, no. 13, pp. 1835-1837, 2002.

[5] Y. K. Chang, "Controllability of impulsive functional differential systems with infinite delay in Banach spaces," Chaos, Solitons and Fractals, vol. 33, no. 5, pp. 1601-1609, 2007.

[6] M. Li, M. Wang, and F. Zhang, "Controllability of impulsive functional differential systems in Banach spaces," Chaos, Solitons and Fractals, vol. 29, no. 1, pp. 175-181, 2006.

[7] J. Jeong, J. Kim, and H. Roh, "Controllability for semilinear retarded control systems in Hilbert spaces," Journal of Dynamical and Control Systems, vol. 13, no. 4, pp. 577-591, 2007.

[8] Z. Tai and X. Wang, "Controllability of fractional-order impulsive neutral functional infinite delay integrodifferential systems in Banach spaces," Applied Mathematics Letters, vol. 22, no. 11, pp. 1760-1765, 2009. 
[9] R. Sakthivel and E. R. Anandhi, "Approximate controllability of impulsive differential equations with state-dependent delay," International Journal of Control, vol. 83, no. 2, pp. 387-393, 2010.

[10] C. G. Gal, "Semilinear abstract differential equations with deviated argument," Research on Evolution Equation Compendium, vol. 333, no. 16, pp. 381-386, 2009.

[11] D. N. Pandey, A. Ujlayan, and D. Bahuguna, "On nonlinear abstract neutral differential equations with deviated argument," Nonlinear Dynamics and Systems Theory, vol. 10, no. 3, pp. 283294, 2010.

[12] J. C. Chang and H. Liu, "Existence of solutions for a class of neutral partial differential equations with nonlocal conditions in the $\alpha$-norm," Nonlinear Analysis: Theory, Methods \& Applications, vol. 71, no. 9, pp. 3759-3768, 2009.

[13] K. Naito, "Controllability of semilinear control systems dominated by the linear part," SIAM Journal on Control and Optimization, vol. 25, no. 3, pp. 715-722, 1987.

[14] A. Pazy, Semigroups of Linear Operators and Appli cations to Partial Differential Equations, vol. 44 of Applied Mathematical Sciences, Springer, New York, NY, USA, 1983. 


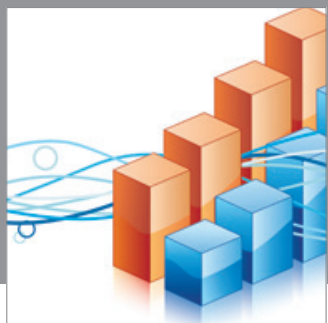

Advances in

Operations Research

mansans

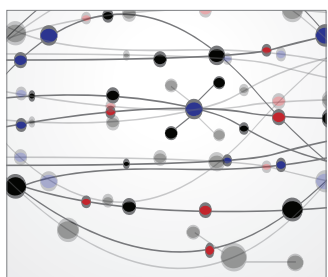

The Scientific World Journal
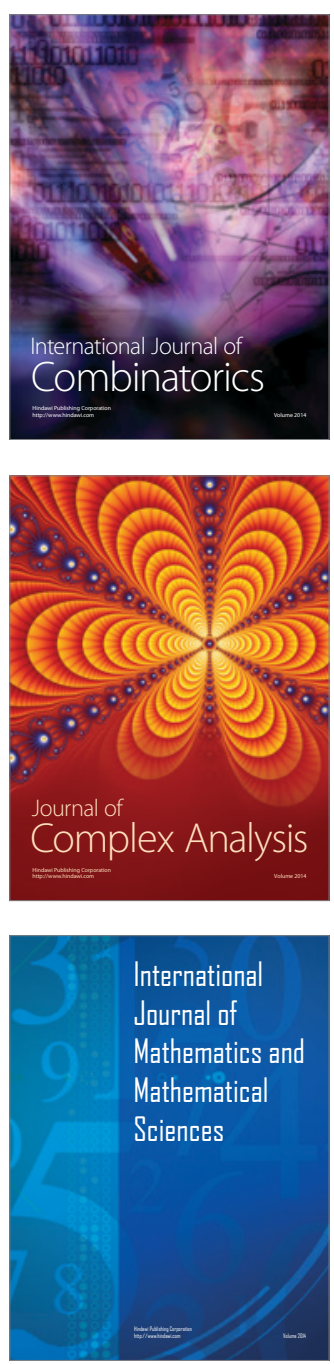
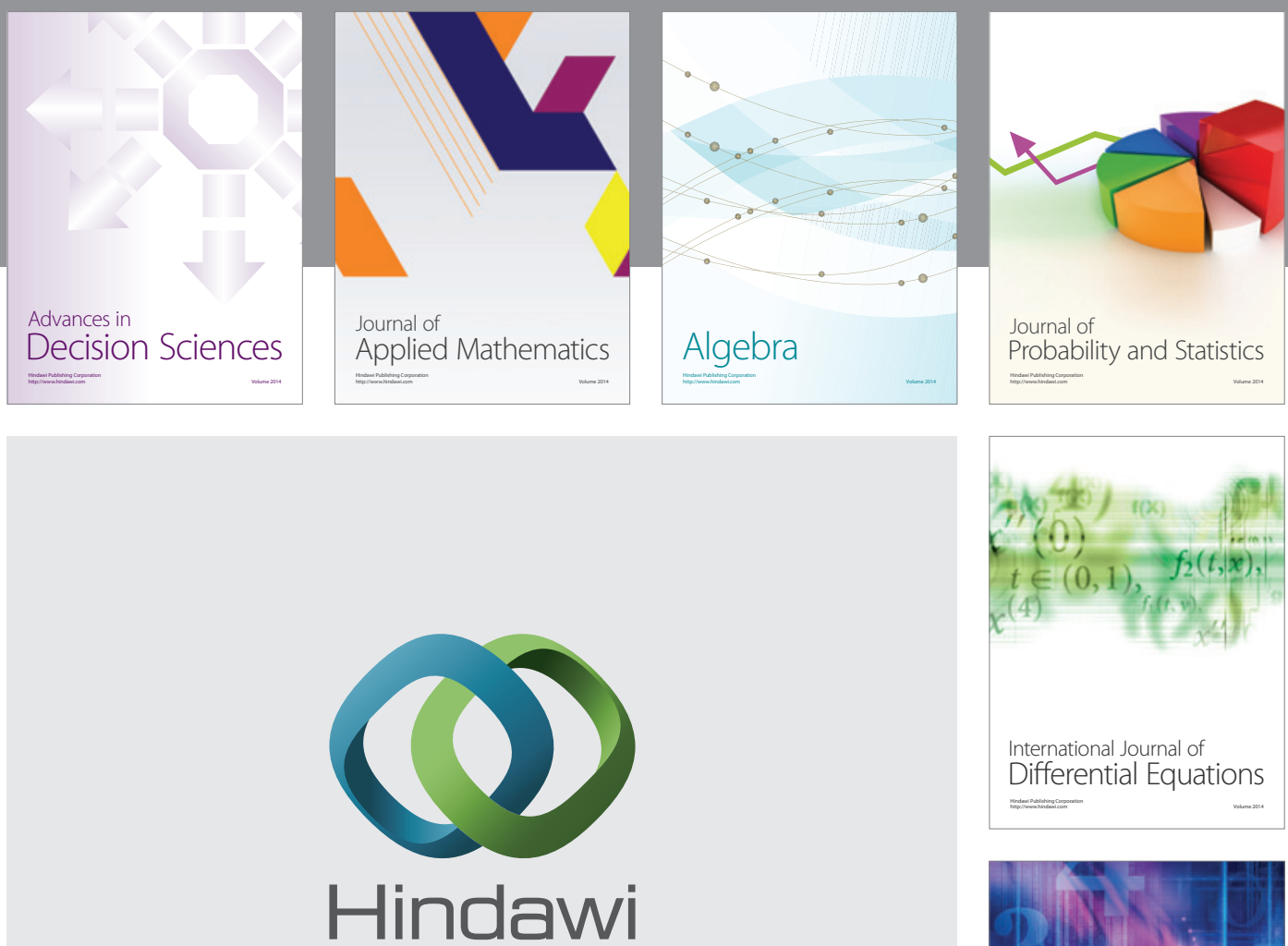

Submit your manuscripts at http://www.hindawi.com
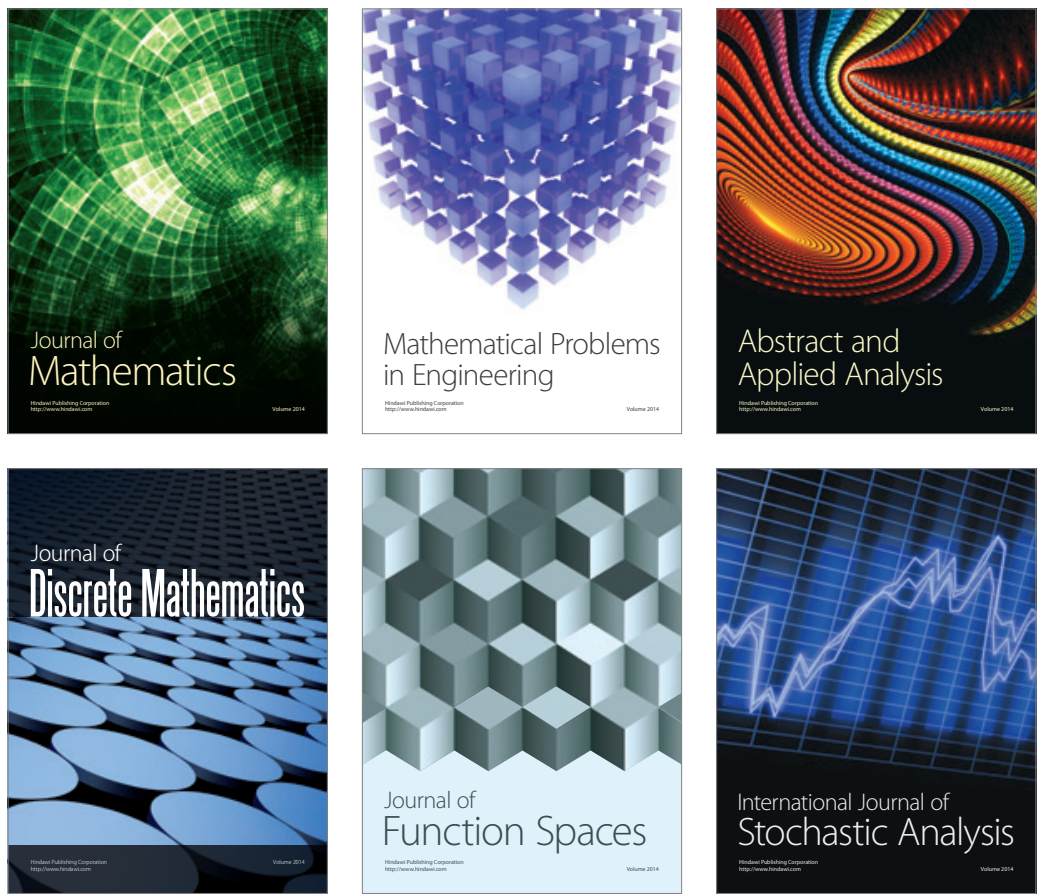

Journal of

Function Spaces

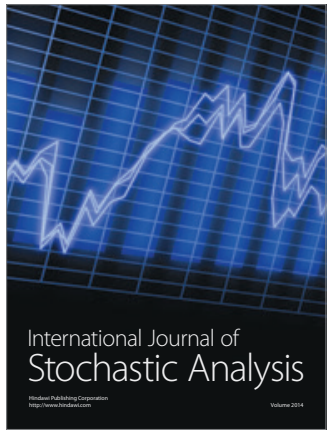

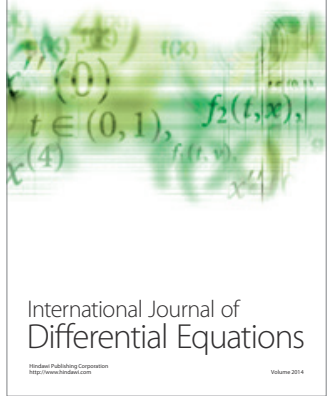
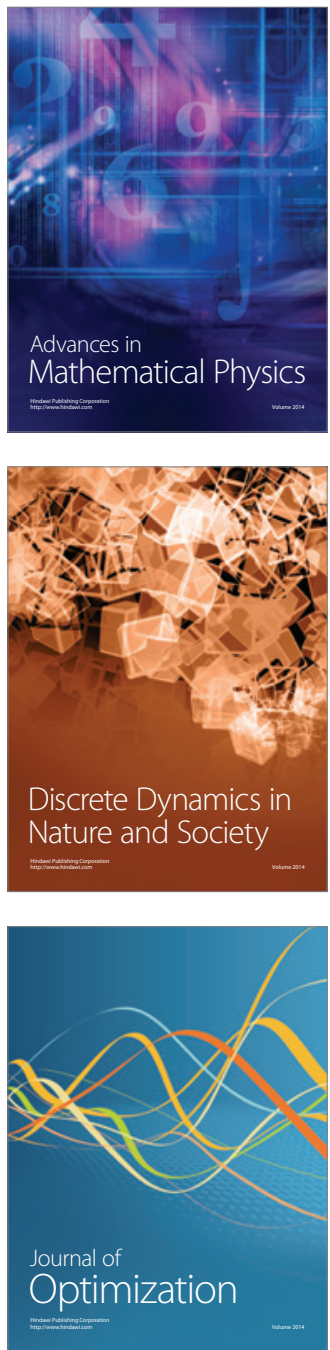